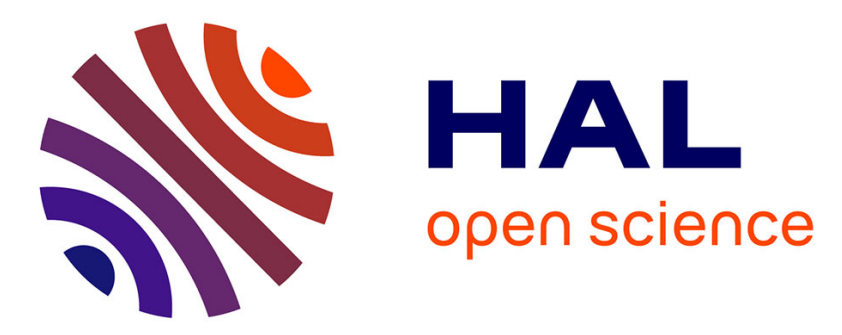

\title{
Lower detection limit of the acousto-optic effect using Optical Feedback Interferometry
}

Einar Knudsen, Julien Perchoux, Thierry Mazoyer, Francis Jayat, Clément Tronche, Thierry Bosch

\section{- To cite this version:}

Einar Knudsen, Julien Perchoux, Thierry Mazoyer, Francis Jayat, Clément Tronche, et al.. Lower detection limit of the acousto-optic effect using Optical Feedback Interferometry. 2020 IEEE International Instrumentation and Measurement Technology Conference (I2MTC), May 2020, Dubrovnik, Croatia. pp.1-4, 10.1109/I2MTC43012.2020.9128405 . hal-02949395

\section{HAL Id: hal-02949395 \\ https://hal.laas.fr/hal-02949395}

Submitted on 25 Sep 2020

HAL is a multi-disciplinary open access archive for the deposit and dissemination of scientific research documents, whether they are published or not. The documents may come from teaching and research institutions in France or abroad, or from public or private research centers.
L'archive ouverte pluridisciplinaire HAL, est destinée au dépôt et à la diffusion de documents scientifiques de niveau recherche, publiés ou non, émanant des établissements d'enseignement et de recherche français ou étrangers, des laboratoires publics ou privés. 


\section{Lower detection limit of the acousto-optic effect using Optical Feedback Interferometry}

\author{
$1^{\text {st }}$ Einar Knudsen \\ LAAS-CNRS \\ Université de Toulouse, INP \\ Toulouse, France \\ einar.knudsen@acoem.com \\ $5^{\text {th }}$ Clément Tronche \\ LAAS-CNRS \\ Université de Toulouse, INP \\ Toulouse, France \\ clement.tronche@laas.fr
}

\author{
$2^{\text {nd }}$ Julien Perchoux \\ LAAS-CNRS \\ Université de Toulouse, INP \\ Toulouse, France \\ julien.perchoux@1aas.fr
}

\author{
$3^{\text {rd }}$ Thierry Mazoyer \\ Dept. of Innovation \\ ACOEM \\ Limonest, France \\ thierry.mazoyer@acoem.com
}

\author{
$6^{\text {th }}$ Thierry Bosch \\ LAAS-CNRS \\ Université de Toulouse, INP \\ Toulouse, France \\ thierry.bosch@laas.fr
}

\author{
$4^{\text {th }}$ Francis Jayat \\ LAAS-CNRS \\ Université de Toulouse, INP \\ Toulouse, France \\ francis.jayat@1aas.fr
}

\begin{abstract}
Measurement and 3D imaging of acoustic waves through the acousto-optic effect has recently been demonstrated by means of Optical Feedback Interferometry (OFI). In this paper we study experimentally the lower limits of detection of an acoustic wave using an OFI sensor. We show that the OFI sensor exhibits a linear response to acoustic power variations, and we obtain a lower limit of detection of $83 \mathrm{~dB}$ rms for a planar acoustic wave at $3 \mathrm{kHz}$. We also determine the equivalent displacement, that is seen by the OFI sensor at this pressure level, to be $96 \mathrm{pm}$. A deeper understanding of the limits of the technology and the quantification of the acousto-optic effect shall help improve the applications already created for the measurement of acoustic pressure waves using OFI.
\end{abstract}

Index Terms-Optical feedback, interferometry, self-mixing, acousto-optic effect.

\section{INTRODUCTION}

Optical Feedback Interferometry (OFI) is a non-contact, high-precision sensing scheme which is applied to many domains of metrology. Today we find OFI in applications such as flowmetry, absolute distance measurements, vibrometry and velocimetry [1]-[6]. Compared to other non-contact sensing technologies, OFI is self-aligned, low cost and robust. Recent applications have applied the sensing scheme to the imaging of acoustic waves through the acousto-optic effect. Bertling et al [6] and Urgiles et al [7] create visual representations of standing and propagating sound waves in air, while Dubois et al proposed an exciting application to image pressure waves in acoustic metamaterials [8].

In these applications they measure the minute changes in the optical path between the laser and the target, through the modulation of the refractive index of air caused by an acoustic pressure wave. These changes in optical path are usually far below the half wavelength of the laser and close to the limitations of the technology. One particular challenge that has not yet been treated is the quantification of the air-pressure change in an acoustic wave using this method.
OFI operates by backscattering the laser light off a target, inducing coherent mixing of the returning light with the laser inner cavity field. The laser wave phase and amplitude is perturbed, and these perturbations are measured by the laser's in-package photodiode. The signal provides us with information about the target the laser is pointing on. If the target is in movement, interferometric fringes are observed. A fringe appears when the target has moved a distance of half the laser's wavelength $\lambda[1],[5],[9]$.

Measurements inferior to the half wavelength of the laser has been reported. Currently the state of the art using the OFI sensing scheme is measurements of $100 \mathrm{pm}$ [2], [10], [11]. Through the acousto-optic effect we measure very small changes in the optical path through the modulation of the refractive index of air in the laser beam's path. The effect has been depicted [6], [7] but there are no evidence of quantification of the acoustic amplitude or the effect on the refractive index so far.

The refractive index of air influences the time it takes for light to travel from the laser to the target and back. P. E. Ciddor [12] made an an exhaustive model to precisely calculate the refractive index of light under given conditions. His model operates in the visible and near infrared, even for small pressure variations.

In this paper we study the dynamic range of an OFI system measuring acoustic signals using the acousto-optic effect. Furthermore, we present a model for the conversion of measured acoustic pressure through the acousto-optic effect to an equivalent displacement in order to quantify the phenomenon. In earlier studies [6], [7], pressure and frequency are controlled, but the waves undergo a free field spherical propagation. To quantify the acousto-optic effect, we have chosen to work with planar waves, where the advantages of such waves are described in sections II-A and II-B. 


\section{THEORY}

\section{A. OFI model and governing equations}

The laser power under external feedback $P_{\mathrm{F}}$ is calculated through the well established equation

$$
P_{\mathrm{F}}=P_{0}\left[1+m \cos \left(\omega_{\mathrm{F}} \tau_{\mathrm{ext}}\right)\right],
$$

where $P_{0}$ is the free running laser power, $m$ is a modulation index that depends on the target reinjection efficiency, and $\omega_{\mathrm{F}}$ is the laser frequency under feedback [1], [5], [7]. An acoustic wave is a variation of air pressure with a subsequent change in refraction index $n$ [12]. Considering a change in $n$, the photon round-trip time in the external cavity $\tau_{\text {ext }}$ is expressed as

$$
\tau_{\text {ext }}=\tau_{0}+\delta \tau
$$

with $\tau_{0}=2 n_{0} L_{\text {ext }} / c$ where $c$ is the speed of light, $n_{0}$ is the refractive index of air without acoustic excitation and

$$
\delta \tau=\int_{0}^{L_{\mathrm{ext}}} \frac{2 \delta n(z)}{c} d z
$$

where $z$ is the coordinate along the laser beam. If we consider a plane pressure wave propagating in a perpendicular direction to the laser beam, the refractive index is constant along the $z$ axis and we can express (3) as

$$
\delta \tau(t)=2 L_{\mathrm{ext}} \delta n(t) / c
$$

where $\delta n(t)=\delta n_{\max } \sin \left(\omega_{\mathrm{a}} t\right)$, with $\omega_{\mathrm{a}}=2 \pi f_{\mathrm{a}}$ where $f_{\mathrm{a}}$ is the acoustic frequency, and $\delta n_{\max }$ is the amplitude of the refractive index modulation of air in a given acoustic wave.

\section{B. Refracive index of air}

The round-trip time $\tau_{\text {ext }}$ in (1) is modulated through the change in $n$ which is in turn modulated by the change of density of air under the effect of the acoustic pressure wave. To calculate variations of $n$ we use Ciddor's model [12]. The model includes wavelength, pressure, temperature, relative humidity and $\mathrm{CO}_{2}$ concentration. Since the acoustooptic induced change in the optical path produces the same effect as a change in physical path, to quantify the acoustooptic effect we calculate an equivalent displacement from the change in refractive index

$$
\delta d_{\mathrm{eq}}=L_{\mathrm{ext}}\left(\frac{\partial n}{\partial p}\right)_{\lambda, P_{0}, \mathrm{~T}_{0}, \mathrm{RH}_{0}, \mathrm{R}_{\mathrm{CO} 2,0}} \times \delta p
$$

where $\partial n / \partial p$ is the change in refractive index with regards to the change in pressure, and $\delta p$ is the pressure difference in an acoustic wave, measured in $\mathrm{Pa} . \lambda$ is the laser wavelength, $P_{0}$ the initial pressure, $T_{0}$ the temperature in Celsius, $\mathrm{RH}$ the relative humidity and $R_{\mathrm{CO}_{2}, 0}$ the $\mathrm{CO}_{2}$ concentration.

The $\delta n_{\max }$ following (4) is calculated for a pressure change $\delta p$ using Ciddor's model, and the constants $\partial n$ and $\partial p$ are computed. By considering planar waves, the pressure $p$ and hence the refractive index $n$ is evenly distributed through the entire optical axis of the laser.

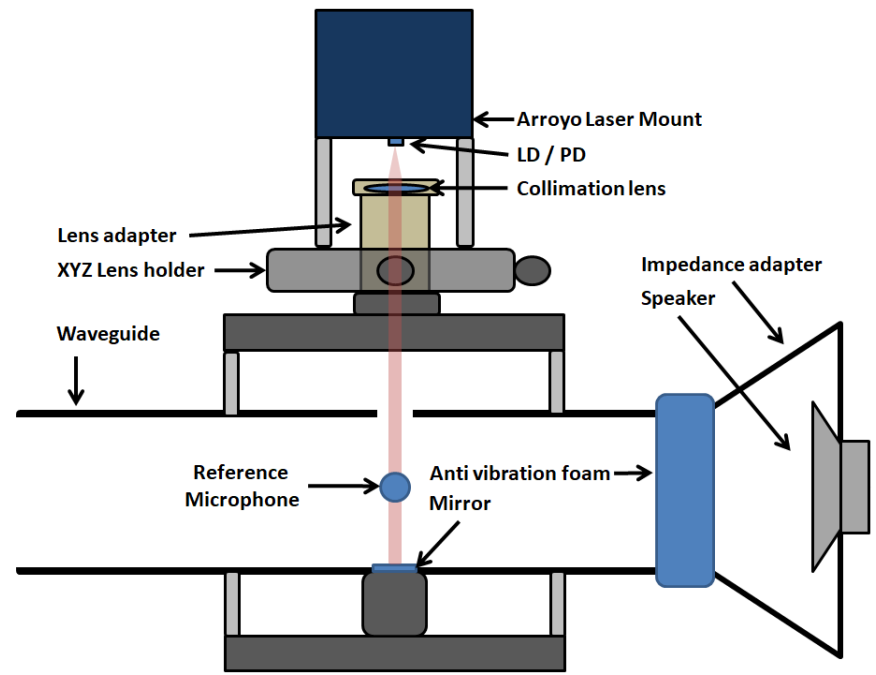

Fig. 1: Top view of experimental setup.

\section{Experiment}

A Distributed Feedback laser diode (1310P5DFB) emitting a transverse and longitudinal single mode at $1310 \mathrm{~nm}$ is mounted on an Arroyo Instruments laser mount. The mount includes a temperature control that is driven by a ThorLabs TED 200C. The laser beam is colimated by a ThorLabs C230TMD-C lens and aimed at a Thorlabs PF05-03-P01 mirror mounted in a Thorlabs KAD12F adjustable mount, $200 \mathrm{~mm}$ from the laser source. The mirror is aligned so that the beam is re-injected into the laser with the best achievable efficiency.

In order to assure planar waves, the acoustic waves are injected into a cylinder of diameter $55 \mathrm{~mm}$ and length $0.5 \mathrm{~m}$. The acoustic wave is generated by a Visatron FRS 8 speaker, driven by a Renkforce SAP-702 amplifier that is mounted inside an acoustic impedance adapter of length $130 \mathrm{~mm}$ at the end of the waveguide. The interferometric system if fixed on the waveguide at $200 \mathrm{~mm}$ from the inlet. A G.R.A.S 46AE metrologic microphone is inserted into the waveguide over the laser beam, flush with the inner wall as to not perturb the pressure wave. The acoustic signal is acquired by an ACOEM 04dB acquisition card connected to the microphone.

The ensemble laser - waveguide - mirror, is placed on vibration absorbing foam to reduce potential vibrations propagating trough the optical table. The impedance adapter and speaker is attached to the waveguide through absorbent foam to reduce vibrations due to the mechanical movement of the speaker membrane. Two PCB 352C18 accelerometers are fixed on the distant sensor parts: one on the laser mount, the other on the mirror mount.

Once acquired, the accelerometer signals are integrated two times before being subtracted from one another to compute the relative displacement.

To determine the linearity and minimal detection level of the system, a continuous sinusoidal acoustic signal with frequency $3 \mathrm{kHz}$ is sent through the waveguide and the OFI signal is recorded for signal amplitudes ranging from $70 \mathrm{~dB}_{\mathrm{rms}}$ 


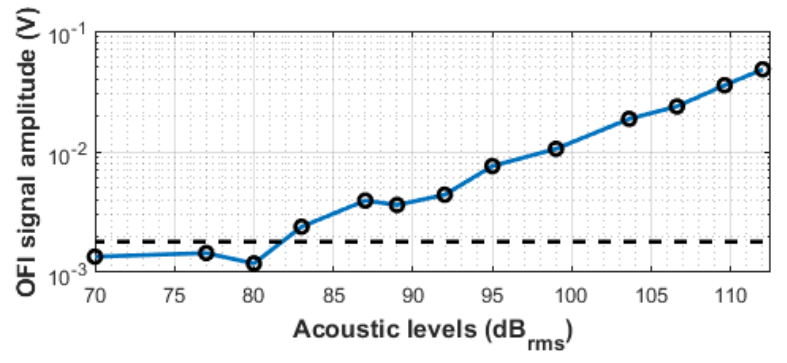

Fig. 2: Blue line: Sensor signal power for acoustic pressures. Dashed black line: Noise level of the sensor.

(0.09 Pa) to $112 \mathrm{~dB}_{\text {rms }}(11.26 \mathrm{~Pa})$. The signals are acquired and filtered using a $2^{\text {nd }}$ order Butterworth bandpass filter with cutoff frequencies $f_{\mathrm{c}_{1}}=2.7 \mathrm{kHz}$ and $f_{\mathrm{c}_{2}}=3.3 \mathrm{kHz}$. An FFT is calculated for each signal, and the amplitude of the $3 \mathrm{kHz}$ peak is recorded.

\section{Numerical simulations}

Using (5) we can calculate an equivalent displacement for an acoustic pressure wave under a given set of conditions. With an OREGON WMR89 weather station we measure the temperature to be $20^{\circ} \mathrm{C}$ and the relative humidity to be $35 \%$. The atmospheric pressure is $101.325 \mathrm{kPa}$, the laser's wavelength is $1310 \mathrm{~nm}$ and the $\mathrm{CO}_{2}$ quantity is set to $450 \mathrm{ppm}$ as proposed in [12]. We calculate the refractive index $n$ over the pressure range of a class one metrological sonometer (65 $\mathrm{kPa}$ to $110 \mathrm{kPa}$ ) with mentioned parameters as input, and we derivate to obtain $\partial n$ for use in (5). Using the same input parameters we can simulate the variation of pressure in an acoustic wave and calculate the equivalent displacement for any given acoustic power.

\section{DISCUSSION}

In Fig. 2 we observe the system's response to acoustic pressure levels. When we decrease the acoustic power from $70 \mathrm{~dB}_{\mathrm{rms}}$ to $112 \mathrm{~dB}_{\mathrm{rms}}$ the response is fairly linear before flattening out at the bottom. The system's response to acoustic the pressures $87 \mathrm{~dB}_{\mathrm{rms}}-92 \mathrm{~dB}_{\mathrm{rms}}$ remains at a similar level. This is possibly caused by a change in $\omega_{\mathrm{F}}(1)$, due to a perturbation in the laser current, change in laser temperature, or to vibrational perturbations. The lowest detectable acoustic amplitude is $83 \mathrm{~dB}_{\text {rms }}$ which is recorded with an amplitude of $2.4 \mathrm{mV}$ by the OFI system.

Fig. 3 shows the theoretical relationship between the amplitude of a plane acoustic wave in Pascals that passes through the laser beam, and the equivalent displacement in nanometers, as calculated using the model in [12]. Plotted in a dashed blue line in the same figure we observe how the sensor's response the acoustic pressure follows the theoretical curve in a linear fashion.

Using (5) we calculate that a 11.26 Pa zero to peak acoustic pressure wave corresponds to an equivalent displacement of $1.64 \mathrm{~nm}$ zero to peak. The lowest detectable amplitude in our experiment is $0.4 \mathrm{~Pa}$ zero to peak and is calculated to be $58 \mathrm{pm}$ in equivalent displacement zero to peak.

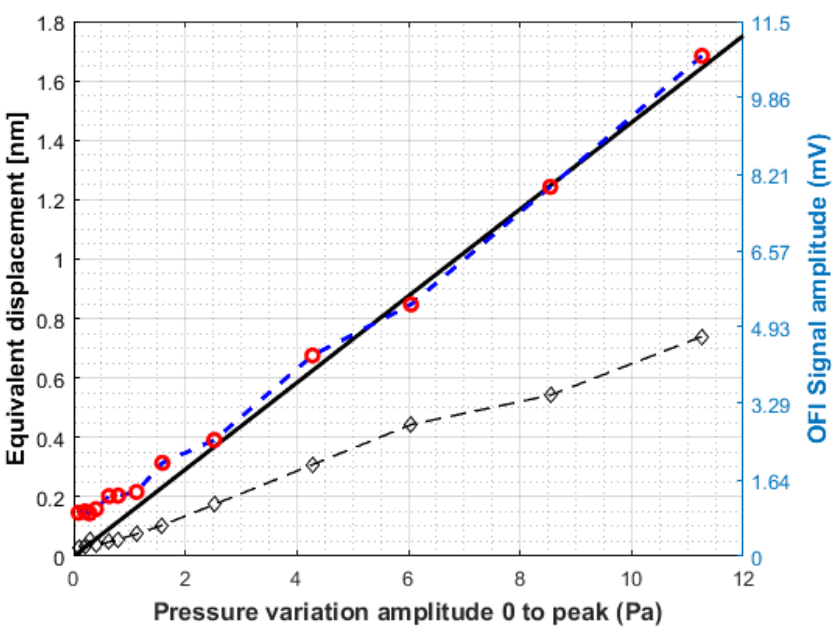

Fig. 3: Black solid line (left axis): Simulated equivalent displacement for given pressure variations. Black striped line (left axis): Differential movement between laser mount and mirror mount for given pressure variations. Blue striped line (Right axis): Sensor response for measured acoustic pressure

For each acoustic power we use the accelerometers to estimate relative displacement between the mirror and the laser. We observe a modulation of the relative distance at the acoustic frequency which is $3 \mathrm{kHz}$. In Fig. 3 we observe how the relative amplitude between the laser mount and the mirror mount descends linearly with the acoustic pressure. When injecting the 11.26 Pa zero to peak pressure wave into the system, we observe a $0.74 \mathrm{~nm}$ zero to peak relative movement between the laser mount and the mirror mount. As such we consider the vibrational levels too strong to be negligible. The average relative movement of the system is $0.038 \mathrm{~nm}$ zero to peak at the lowest detectable acoustic power of $0.4 \mathrm{~Pa}$ zero to peak. Therefore we calculate a new lower limit of detection for the system by adding the measured vibrational displacement to the theoretical equivalent displacement, and we obtain $96 \mathrm{pm}$ zero to peak, that is similar to the lowest reported values for vibration OFI sensing in the literature. Thus we account for the possible perturbation due to vibrations in the system.

\section{CONCLUSION}

We have studied the response of an OFI sensing system measuring the acousto-optic effect for different amplitudes of acoustic pressure variation. A model was established to convert the measured acoustic pressure amplitude to an equivalent displacement. Using planar acoustic waves made it possible to control and accurately measure the pressure $p$ along the optical axis of the laser. The system was designed so that the acoustic waves were completely planar in order to calculate the equivalent displacement using the model.

We have found that there is a linear relationship between the optical power variation and the acoustic pressure variations. Due to vibrations in the system the relative distance between the laser mount and the reflector mount is modulated at the 
acoustic frequency. We have determined the lowest detectable acoustic amplitude detectable, taking into account these perturbations. The lowest detectable optical path variation we are able to detect with our system is $96 \mathrm{pm}$ when the acoustic wave pressure variation is $0.4 \mathrm{~Pa}$. The relative movement due to vibrations is $38 \mathrm{pm}$ and the calculated equivalent displacement is $58 \mathrm{pm}$. In [2] the lower threshold of vibration reported is $0.1 \mathrm{~nm}$, giving our system essentially the same performances. In order to eliminate the effect of the vibrations the experiment is to be improved so that the laser and mirror mounts no longer are in contact with the waveguide. The acoustic generator should be placed on vibration absorbent foam in order to eliminate vibrations propagating through the optical table.

A future perspective is to continue the development of our model to make it compatible with spherical free-field acoustic waves. Such a model would be of great advantage when studying the acousto-optic effect using OFI, and could even be applied to previous studies already published. In doing so we could, using OFI, create a non-contact, cost effective and accurate measurement instrument for the quantification of freefield acoustic pressure waves. As the instrument would be noncontact, the wave would not be perturbed as it is measured.

\section{ACKNOWLEDGMENTS}

This work has been performed with the LabCom CapIRO a joint laboratory with the Toulouse INP, The LAAS - CNRS and ACOEM. Grants and funding provided by the Agence Nationale de la Recherche (ANR) the Association Nationale Recherche Technologie (ANRT).

\section{REFERENCES}

[1] T. Taimre, M. Nikolić, K. Bertling, Y. L. Lim, T. Bosch, and A. D. Rakić, "Laser feedback interferometry: a tutorial on the self-mixing effect for coherent sensing," Advances in Optics and Photonics, vol. 7, p. 570, Sept. 2015.

[2] G. Giuliani, S. Bozzi-Pietra, and S. Donati, "Self-mixing laser diode vibrometer," Measurement Science and Technology, vol. 14, pp. 24-32, Jan. 2003.

[3] R. Atashkhooei, E. E. Ramírez-Miquet, R. d. C. Moreira, A. Quotb, S. Royo, and J. Perchoux, "Optical Feedback Flowmetry: Impact of Particle Concentration on the Signal Processing Method," IEEE Sensors Journal, vol. 18, pp. 1457-1463, Feb. 2018.

[4] A. L. Arriaga, F. Bony, and T. Bosch, "Real-Time Algorithm for Versatile Displacement Sensors Based on Self-Mixing Interferometry," IEEE Sensors Journal, vol. 16, pp. 195-202, Jan. 2016.

[5] M. Veng, J. Perchoux, and F. Bony, "Fringe Disappearance in SelfMixing Interferometry Laser Sensors: Model and Application to the Absolute Distance Measurement Scheme,' IEEE Sensors Journal, pp. 1$1,2019$.

[6] K. Bertling, J. Perchoux, T. Taimre, R. Malkin, D. Robert, A. D. Rakić, and T. Bosch, "Imaging of acoustic fields using optical feedback interferometry," Optics Express, vol. 22, p. 30346, Dec. 2014.

[7] P. F. Urgiles Ortiz, J. Perchoux, A. L. Arriaga, F. Jayat, and T. Bosch, "Visualization of an acoustic stationary wave by optical feedback interferometry," Optical Engineering, vol. 57, p. 1, Feb. 2018.

[8] M. Dubois, J. Perchoux, A. L. Vanel, C. Tronche, Y. Achaoui, G. Dupont, K. Bertling, A. D. Rakić, T. Antonakakis, S. Enoch, R. Abdeddaim, R. V. Craster, and S. Guenneau, "Acoustic flat lensing using an indefinite medium," Phys. Rev. B, vol. 99, p. 100301, Mar 2019.

[9] R. Lang and K. Kobayashi, "External optical feedback effects on semiconductor injection laser properties," IEEE Journal of Quantum Electronics, vol. 16, pp. 347-355, Mar. 1980.

[10] M. Kossl and I. J. Russell, "Basilar membrane resonance in the cochlea of the mustached bat.," Proceedings of the National Academy of Sciences, vol. 92, pp. 276-279, Jan. 1995.
[11] A. N. Lukashkin, M. E. Bashtanov, and I. J. Russell, "A selfmixing laser-diode interferometer for measuring basilar membrane vibrations without opening the cochlea," Journal of Neuroscience Methods, vol. 148 , pp. 122-129, Oct. 2005.

[12] P. E. Ciddor, "Refractive index of air: new equations for the visible and near infrared," Appl. Opt., vol. 35, pp. 1566-1573, Mar 1996. 